\title{
Severe glomerular C3 deposition indicated severe renal lesions and poor prognosis in patients with Immunoglobulin A Nephropathy
}

\author{
jianliang $\mathrm{wu}^{1}$, Zhizhi $\mathrm{Hu}^{1}$, Yuxi Wang ${ }^{1}$, Danni $\mathrm{Hu}^{1}$, Qian Yang ${ }^{1}$, Yueqiang $\mathrm{Li}^{1}$, Wei Dai ${ }^{1}$, \\ Fengming Zhu ${ }^{1}$, Juan Yang ${ }^{1}$, Meng Wang ${ }^{1}$, Han Zhu ${ }^{1}$, Liu Liu ${ }^{1}$, Xiaofeng He ${ }^{1}$, Min Han ${ }^{1}$, \\ Ying $\mathrm{Yao}^{1}$, Guangchang $\mathrm{Pei}^{1}$, Rui Zeng ${ }^{1,2}$, and Gang $\mathrm{Xu}^{1}$ \\ ${ }^{1}$ Tongji Hospital of Tongji Medical College of Huazhong University of Science and \\ Technology \\ ${ }^{2}$ Huazhong University of Science \& Technology
}

July 7, 2020

\begin{abstract}
ABSTRACT Background and objectives Glomerular C3 deposition is often observed in renal biopsies of patients with IgA nephropathy (IgAN), however, the relationship between the level of C3 deposition and the long-term prognosis of IgAN have rarely been reported. In this observational cohort study, we aimed to evaluate the prognostic value of glomerular C3 deposition in the progression of IgAN. Materials and Methods From June 2009 to June 2010, a total of 136 adult patients with IgAN were enrolled in the study. Glomerular deposition of the complement protein C3, immune complexes, and inflammatory cells were detected in renal biopsy tissue. The level of renal lesions, glomerular immune complexes, and the density of interstitial inflammatory cells were analyzed. Based on an average of 105 months of follow-up, the predictive value of glomerular C3 deposition for IgAN progression was investigated. Results Of the 136 patients, 102 patients were classified into the glomerular C3low group and 34 patients were classified into the glomerular C3high group. Patients in C3high group had more immune complexes deposition than those in C3low group. In C3high group, the degree of renal lesion and interstitial fibrosis were severer compared to C3low group. Renal biopsy in C3high group presented with higher density of interstitial inflammatory cells that in C3low group. The density of glomerular C3 deposition was associated with poor renal survival over a 105-month follow-up. Conclusions The high density of glomerular C3 deposition was associated with severity of renal lesions and predicted a long-term poor renal survival for IgAN patients.
\end{abstract}

\section{INTRODUCTION}

IgA nephropathy (IgAN), characterized by predominant IgA deposition in the glomerular mesangium, is the most common form of primary glomerulonephritis worldwide[1, 2]. The other histological features of IgA nephropathy are mesangial cell proliferation with complement 3 (C3) and variable IgG and / or IgM co-deposits[3]. IgAN presented with a wide range of histology and clinical features, among which about one-third of patients develop into ESRD within 25 years $[1,4,5]$. As no specific treatment is available for IgAN, it is particularly important to find the potential risk factors for the long-term prognosis of IgAN. It is well established that risk factors for the progression of IgAN include the presence of proteinuria, high blood pressure, and declined eGFR[6, 7]. The MEST scoring system including mesangium hypercellularity (M), endocapillary hypercellularity (E), segmental glomerulosclerosis (S), and tubular atrophy/interstitial fibrosis $(\mathrm{T})$, was also validated in a variety of studies for the prediction of clinical outcomes[8, 9]. T cell subpopulations were also identified to be associated with the severity of proteinuria and hematuria, elevated serum creatinine levels and reduced GFR in IgAN[10].

Glomerular co-deposition of C3 and IgA1 was observed in at least 90\% of IgAN renal biopsies[3, 11]. Previous 
studies have shown that the pathogenic co-deposition of C3 and IgA1 induces proliferation of mesangial cells and secretion of cytokines and extracellular matrix, which plays a key role in the pathogenesis of $\operatorname{IgAN}[12$, 13]. However, the role of complement activation on the progression and prognosis of IgAN remains unclear. Although renal biopsy remains the gold standard diagnosis, recent efforts have been made to find new biomarkers that might predict progression and prognosis of IgAN.

In this observational cohort study, we analyzed clinical features according to the pathologic findings and glomerular immune complex deposition and then investigated the predictive value of glomerular C3 deposition in predicting the prognosis of patients with IgAN.

\section{MATERIALS AND METHODS}

\section{Patients}

The study was carried out in accordance with the Declaration of Helsinki and approved by Committee on Research Ethics of Tongji Hospital, Huazhong University of Science and Technology. Renal biopsy was performed in 205 Chinese adult patients aged between 18 and 70 years with IgAN between June 2009 to June 2010. All patients met the diagnostic criteria for IgAN, which was published by the Oxford classification working group [14]. Patients were excluded if they had received glucocorticoids or immunosuppressants treatment before renal biopsy; if they had HBV infection or liver chirosis; if they had a concomitant autoimmne disease ; or if they did not have complete follow-up data. 136 patients were eventually enrolled into this study (Figure 1).

\section{Clinical studies and laboratory data}

The following parameters were collected at the time of the renal biopsy, including gender, age, blood pressure, hematuria, proteinuria, serum uric acid, serum albumin, blood urea nitrogen, serum creatinine and eGFR. To assess the presence of hematuria, $10 \mathrm{ml}$ of clean fresh urine that came from the middle piece was centrifuged for 5 minutes at $1500 \mathrm{rpm} ; 0.2 \mathrm{ml}$ of sediment was taken and observed under microscopy. The severity of erythrocyturia was characterized as follows: $>20 \mathrm{RBC} / \mathrm{HPF}$ as $3+$. The estimated glomerular filtration rate (eGFR) was calculated using the CKD-epidemiology collaboration (CKD-EPI) formula: eGFR (ml/min per1.73 m2) $=186 \times \mathrm{Scr}^{-1.154} \times$ age $^{-0.203} \times 0.742($ if female $) \times 1.227$

\section{Histologic Evaluation}

The biopsy specimens were processed for light microscopy, immunofluorescence and electron microscopy. Paraffin sections for light microscopy were stained with hematoxylin and eosin, periodic acid-Schiff, and Masson's trichrome stain for pathologic analysis. Direct immunofluorescence for IgA, IgG, IgM, and C3 was performed in frozen sections. Clinical renal pathologists performed qualitative assessment of the degree of immunofluorescent staining. The degree of glomerular C3 immunofluorescent stain intensity was reported on a scale of $1+$ to $3+$, with $1+$ representing low-intensity staining; $2+$, medium-intensity staining; or $3+$, high-intensity staining. Glomerular IgG and IgM deposition were graded by absent (score, 0), mild (score, 1), marked (score, 2), respectively. Glomerular $\operatorname{IgA}$ deposition were graded by $\operatorname{IgA}=2+$ and $\operatorname{IgA}>2+$. Pathological features were scored using the existing literature $[14,15]$. In particular, mesangial hypercellularity, segmental glomerulosclerosis, endocapillary hypercellularity, tubular atrophy/interstitial fibrosis were graded by Oxford classification. For each biopsy, we determined the fraction of glomeruli with cellular or fibrocellular or fibrous crescents. A crescent was defined as extracapillary proliferation of more than two cell layers of any size; this definition was uniform in all studies. The severity of global glomerulosclerosis and arterial wall thickening was graded in three groups: absent (score, 0), mild (score, 0), and marked (score, 0). Crescents and arterial hyalinosis were scored as either absent or present.

\section{Immunohistochemistry and immunofluorescence}

Immunohistochemical staining was performed on $3-\mu \mathrm{m}$ paraffinized sections of renal tissue. Detection of CD68, CD4, CD8 and CD20 (Maxim-Bio), tryptase and CD1c (Abcam) was performed on paraffin sections by an immunohistochemical kit (DAKO, Santa Clara, USA) according to the manufacturer's instructions. 
For immunofluorescence, the primary antibody against C3, IgA, IgG, and IgM (Gene-Bio) and Alexa Flour 488-conjugated secondary antibody (Jackson ImmunoRearch, West Grove, USA) were used. The numbers of each subset of interstitial inflammatory cells were counted under five equivalent high-power cortical fields $(\mathrm{HPFs})(\times 400)$ and were expressed as the average number of cells per square millimeter [16]. The labeled cells were counted three times per high power field (HDF) to reduce counting errors, and the average number was recorded as the density of positive cells in this renal section.

\section{Evaluation of outcomes}

For the present analysis, we considered the creatinine doubling from baseline as the primary endpoint; secondary endpoint was ESRD, which was defined as initiation of renal replacement therapy including permanent hemodialysis, peritoneal dialysis, or renal transplantation.

\section{Statistical Analyses}

Statistical analyses were performed using SPSS 13.0 software (SPSS Inc., Chicago, Illinois, USA). The Kolmogorov-Smirnov test was used to analyze the normality of the distribution of parameters. Continuous variables are expressed mean $\pm \mathrm{SD}$, while categorical variables are expressed as a number (percentage). Comparison between 2 groups using the t test or chi-squared test. Nonparametric variables are described as medians and quartile ranges, and the comparisons between groups were made using

the Mann-Whitney test or the Kruskal-Wallis test. Patients were classified into C3 ${ }^{\text {low }}$ and $\mathrm{C} 3^{\text {high }}$ groups by grading the density of glomerular C3 deposition. The descriptive analysis of the time reaching the primary and secondary endpoint was performed by computing survival curves according to the Kaplan-Meier method, and between-group survival was compared by the log-rank test. Univariate and multivariate Cox proportional hazards models were used to determine the factors associated with the risk of reaching the creatinine doubling from baseline (multifailure model) or ESRD. Results were expressed as hazard-rate (HRs) with CI. Univariate and multivariate linear regression analyses were used to determine the associations between the density of glomerular C3 deposition and other variables that correlate with renal outcome. The multivariate models were built considering that the main interest was the identification of prognostic factors, present at the enrollment and statistically significant at univariate analysis. Two-sided $\mathrm{P}$ values $<0.05$ were considered statistically significant.

\section{Results}

Demographic and clinical characteristics according to the degree of glomerular C3deposition at the time of biopsy

According to the scheme, 136 patients were enrolled and followed up 105 months after renal biopsy. The cohort of 136 patients with IgAN was stratified according to C3 deposition on biopsy specimens as either glomerular $\mathrm{C}^{\text {low }}$ IgAN (C3 score of $1+$ and $2+$ on immunofluorescent staining, $n=102$ ) or glomerular C3 $3^{\text {high }}$ IgAN (C3 score of $3+$ on immunofluorescent staining, $n=34$ ) (Figure 1). We further discovered that patients in $\mathrm{C} 3^{\text {high }}$ group suffered from higher percentage of glomerular IgA, IgM and IgG deposition (Figure 2).

The baseline characteristics of the study population was presented with no significant differences between glomerular C $3^{\text {low }}$ group and glomerular C $3{ }^{\text {high }}$ group in terms of sex, age, serum C3, blood pressure, erythrocyturia, serum uric acid, serum albumin or blood urea nitrogen (Table 1). At time of biopsy for the overall cohort $(\mathrm{n}=136)$, the median degree of proteinuria was $1.0 \mathrm{~g} / 24 \mathrm{~h}$ and the mean level of serum creatinine was $77.6(\mu \mathrm{mol} / \mathrm{L})$. Patients with $\mathrm{C} 3^{\text {high }} \mathrm{IgAN}$ compared to $\mathrm{C} 3^{\text {low }} \mathrm{IgAN}$ had a higher serum creatinine $\left(\mathrm{C} 3^{\text {high }}\right.$ : 89.4 vs. $\mathrm{C} 3^{\text {low }}: 74.9 \mu \mathrm{mol} / \mathrm{L}, \mathrm{P}=0.008$ ), and a higher degree of proteinuria $\left(\mathrm{C} 3^{\text {high }}: 1.6\right.$ vs. C $^{\text {low }}: 0.9$ $\mathrm{g} / 24 \mathrm{~h}, \mathrm{P}<0.001)$. The estimated glomerular filtration rate $(76.0$ vs. $100.8 \mathrm{ml} / \mathrm{min}$ per1.73 $\mathrm{m} 2, \mathrm{P}=0.001)$ was lower in $\mathrm{C} 3^{\text {high }}$ group (Table 1).

IgAN patients in $\mathrm{C3}^{\text {high }}$ group presented with severe pathological lesions and suffered more interstitial inflammatory cell infiltration 
In consistent with the higher proteinuria level and lower eGFR, IgAN patients in $\mathrm{C} 3^{\text {high }}$ group presented with a higher percentage of mesangial hypercellularity ( $56.9 \%$ versus $27.5 \%$; $\mathrm{P}=0.003)$, segmental glomerulosclerosis (41.2\% versus $19.6 \% ; \mathrm{P}=0.012$ ), severe tubular atrophy/interstitial fibrosis (T2, $44.1 \%$ versus $17.6 \%$; $\mathrm{P}=0$. 002), crescents $(52.9 \%$ versus $33.3 \% ; \mathrm{P}=0.042)$, severe arterial wall thickening $(29.4 \%$ versus $12.7 \%$; $\mathrm{P}=0.034)$, severe arterial hyalinosis $(47.1 \%$ versus $21.6 \% ; \mathrm{P}=0.004)$ (Table 2$)$. The two groups showed no difference in endocapillary hypercellularity or global glomerulosclerosis. Representative photographs of light microscopy (PAS and Masson) and electron microscopy of the two groups were shown in Figure 3.

To understand inflammatory cell infiltration in these patients, we detected CD68+ macrophages, CD4+, CD8+ T lymphocytes, tryptase+ mast cells, CD20+ B lymphocytes and CD1c+ myeloid dendritic cells in renal biopsies. We found that patients in $\mathrm{C} 3^{\text {high }}$ group presented with intense interstitial macrophages and $\mathrm{T}$ lymphocytes infiltration than that in $\mathrm{C} 3^{\text {low }}$ group (Figure 4). Renal tissues were revealed more interstitial tryptase + mast cells infiltration in the $\mathrm{C}^{\text {high }}$ group in renal biopsies of IgAN patients rather than CD20+ B lymphocytes and CD1c+ myeloid dendritic cells (Supplementary figure).

\section{Prognostic value of glomerular C3 deposition for renal outcome in patients with IgAN}

During a follow-up of 105 months, the primary endpoint of the creatinine doubling from baseline occurred in $22.1 \%(30 / 136)$ patients. Survival curves were performed according to the Kaplan-Meier method. Proportion of renal survival (absence of creatinine doubling) were $83.3 \%(85 / 102)$ v.s. $61.8 \%(21 / 34)$ in C3 ${ }^{\text {low }}$ and C ${ }^{\text {high }}$ group at the end of 105-month follow-up, respectively (Figure 5A). Estimated overall survival time were 97.8 months for patients in $\mathrm{C} 3^{\text {low }}$ group; 81.9 months for patients in C3 ${ }^{\text {high }}$ group, $P=0.025$ (Figure 5A). The secondary endpoint of ESRD occurred in 13.2\% (18/136) patients. Proportion of renal survival (absence of ESRD) were $93.1 \%(95 / 102)$ v.s. $67.6 \%(23 / 34)$ in $\mathrm{C} 3^{\text {low }}$ and $\mathrm{C} 3^{\text {high }}$ group, respectively. Estimated overall survival time were 100.2 months for patients in $\mathrm{C} 3^{\text {low }}$ group; 86.5 months for patients in $\mathrm{C} 3{ }^{\text {high }}$ group, $\mathrm{P}=0.002$ (Figure 5B).

We then investigated whether the density of glomerular C3 deposition could independently predict poor renal survival. In a multivariate Cox regression model, renal outcome for doubling of Scr was significantly associated with serum creatinine, proteinuria and density of glomerular C3 deposition (Table 3, model 1). When the degree of Oxford-MEST lesion classification was included in a multivariate model, the higher intensity of glomerular C3 deposits remained as an independent predictor of Scr doubling (model 2). Interestingly, the results of model 3 and model 1 are exactly the same, even though there are many differences between the two variables.

By using a multivariate Cox regression analysis, the incidence for ESRD was significantly associated with serum creatinine, proteinuria and glomerular C3 density at biopsy (Table 3, model 1). When the degree of Oxford-MEST lesion classification was included in a multivariate model, the higher intensity of glomerular C3 deposits remained as an independent predictor for ESRD (model 2).

Different models depending on multivariate analyses revealed that glomerular C3 deposits, when included with clinical variables, or included with Oxford-MEST lesion classification, was an independent predictor of Scr doubling and ESRD (Table 3, 4).

\section{DISCUSSION}

Although the risk factors affecting the poor prognosis of patients with IgAN have been described[17], the most influential factor in endpoint outcomes of patients with IgAN remains unclear. In the present study, we assessed 136 patients with IgAN, including 102 patients in C3 $3^{\text {low }}$ group and 34 patients in C3 ${ }^{\text {high }}$ group, to investigate whether glomerular C3 deposition has the value of evaluating clinical prognosis and predicting kidney outcome according to an average of 105 months of follow-up. We found that glomerular C3 deposition was associated with deterioration of renal function and prognosis in patients with IgAN.

Complement regulatory proteins are expressed on the surface of almost all resident kidney cells[18]. Of all the complement proteins co-deposited with IgA1, C3 is the most abundant. Many studies have previously showed that glomerular C3 deposition and complement activation was involved in pathogenesis of patients with 
various kidney disease including IgAN[19-21], lupus nephritis[22], C3 glomerulopathy[23], and antineutrophil cytoplasmic antibody (ANCA)-mediated crescentic glomerulonephritis[24]. The intensity of glomerular C3 deposition has been negatively correlated with renal survival in a cohort study of 111 kidney transplant recipients with transplant glomerulopathy[25]. Interestingly, extraglomerular C3 deposits also affects clinical outcomes in IgAN patients[26]. The classical, alternative and lectin pathways are three known pathways for complement system activation. Each pathway has its own triggering mechanism; however, once the C3 convertases is created, all pathways will be unified. Thus, glomerular C3 deposition in IgAN highlights the pathophysiological role of activation of complement fragments.

In this study, we found that with patients with heavy glomerular C3 deposition suffered higher proteinuria level and lower eGFR, higher percentage of mesangial hypercellularity, segmental glomerulosclerosis, severe tubular atrophy/interstitial fibrosis, crescents, severe arterial wall thickening, severe arterial hyalinosis. According to the follow-up data, patients in $\mathrm{C} 3^{\text {high }}$ group had a significantly higher risk of Scr doubling and ESRD than those in $\mathrm{C} 3^{\text {low }}$ group, which was consistent with the previous studies. Nam et al. showed that the glomerular C3 and C4d (especially C3) deposition was an independent risk factor for progression of IgAN [27]. Kim et al. found decreased circulating C3 levels and mesangial C3 deposition predicted renal outcome in patients with IgAN according to 53.7 months follow-up. They also showed a remarkable negative relevant relation between serum C3 levels and mesangial C3 deposition [28]. In our study, although we excluded patients who had received glucocorticoids or immunosuppressants treatment before renal biopsy, we did not find the negative relevant relation serum C3 levels and mesangial C3 deposition in IgAN patients. Besides the histopathlogical and clinical parameters, we further found $\mathrm{C} 3^{\text {high }}$ group contained more interstitial macrophages, $\mathrm{T}$ lymphocytes and mast cells infiltration than the $\mathrm{C} 3^{\text {low }}$ group. It suggested glomerular C3 deposition might be related to the activation of local immune response in the progression of IgAN.

There are several limitations of our study. First, the cohort was not large enough. In the future, large prospective studies and repeat biopsy after glucocorticoids therapy of IgAN patients are expected. Second, we cannot dissect individual role of alternative and lectin pathways. Other complement components were not available in our current research.

In conclusion, our study suggested the clinical significance of glomerular C3 deposition in clinical practice and indicated that high density C3 deposition in kidney were associated with severe renal lesions and the activation of local immune response in the progression of IgAN. Based on an average of 105 months of follow-up, glomerular C3 deposition were independently associated with poor renal outcome in patients with IgAN. Thus, these findings suggest that glomerular C3 deposition are potentially served as a predictor factor of poor prognosis in patients with IgAN.

\section{FUNDING}

This work was supported by the National Natural Science Foundation of China for Young Scholar (Grant No.81600556,81700653,81700597,81800608); Major Research plan of the National Natural Science Foundation of China (Grant No. 91742204); International (regional) cooperation and exchange projects, (NSFC-DFG, Grant No. 81761138041); National Natural Sciences Foundation of China (Grants 81470948, 81670633,81570669, 81570615); the National key research and development program (Grants 2016YFC0906103); the National Key Technology R\&D Program (Grant 2013BAI09B06,2015BAI12B07); The third project for the young and middle-aged excellent medical talents of Wuhan.

1. Lai, K.N., et al., IgA nephropathy. Nat Rev Dis Primers, 2016.2 : p. 16001.

2. Jarrick, S., et al., Mortality in IgA Nephropathy: A Nationwide Population-Based Cohort Study. J Am Soc Nephrol, 2019. 30 (5): p. 866-876.

3. Jennette, J.C., The immunohistology of IgA nephropathy. Am J Kidney Dis, 1988. 12 (5): p. 348-52.

4. Wyatt, R.J. and B.A. Julian, IgA nephropathy. N Engl J Med, 2013. 368 (25): p. 2402-14.

5. Walsh, M., et al., Histopathologic features aid in predicting risk for progression of IgA nephropathy. Clin 
J Am Soc Nephrol, 2010.5 (3): p. 425-30.

6. D'Amico, G., Natural history of idiopathic IgA nephropathy and factors predictive of disease outcome. Semin Nephrol, 2004.24 (3): p. 179-96.

7. Huang, L., et al., IgA nephropathy factors that predict and accelerate progression to end-stage renal disease. Cell Biochem Biophys, 2014. 68 (3): p. 443-7.

8. Coppo, R., et al., Validation of the Oxford classification of IgA nephropathy in cohorts with different presentations and treatments.Kidney Int, 2014. 86 (4): p. 828-36.

9. Barbour, S.J., et al., The MEST score provides earlier risk prediction in lgA nephropathy. Kidney Int, 2016. 89 (1): p. 167-75.

10. Ruszkowski, J., et al., T cells in IgA nephropathy: role in pathogenesis, clinical significance and potential therapeutic target.Clin Exp Nephrol, 2019. 23 (3): p. 291-303.

11. Maillard, N., et al., Current Understanding of the Role of Complement in IgA Nephropathy. J Am Soc Nephrol, 2015. 26 (7): p. 1503-12.

12. Novak, J., et al., IgA1-containing immune complexes in IgA nephropathy differentially affect proliferation of mesangial cells.Kidney Int, 2005. 67 (2): p. 504-13.

13. Novak, J., et al., IgA1 immune complexes from pediatric patients with IgA nephropathy activate cultured human mesangial cells.Nephrol Dial Transplant, 2011. 26 (11): p. 3451-7.

14. Working Group of the International Ig, A.N.N., et al., The Oxford classification of IgA nephropathy: pathology definitions, correlations, and reproducibility. Kidney Int, 2009. 76 (5): p. 546-56.

15. Trimarchi, H., et al., Oxford Classification of IgA nephropathy 2016: an update from the IgA Nephropathy Classification Working Group. Kidney Int, 2017. 91 (5): p. 1014-1021.

16. Lin, M., et al., Toll-like receptor 4 promotes tubular inflammation in diabetic nephropathy. J Am Soc Nephrol, 2012.23 (1): p. 86-102.

17. Barbour, S. and H. Reich, An update on predicting renal progression in IgA nephropathy. Curr Opin Nephrol Hypertens, 2018.27 (3): p. 214-220.

18. Thurman, J.M. and C.M. Nester, All Things Complement. Clin J Am Soc Nephrol, 2016. 11 (10): p. 1856-1866.

19. Rizk, D.V., et al., The Emerging Role of Complement Proteins as a Target for Therapy of IgA Nephropathy. Front Immunol, 2019.10 : p. 504.

20. Liu, L., et al., C3a, C5a renal expression and their receptors are correlated to severity of IgA nephropathy. J Clin Immunol, 2014.34 (2): p. 224-32.

21. Paunas, T.I.F., et al., Glomerular abundance of complement proteins characterized by proteomic analysis of laser-captured microdissected glomeruli associates with progressive disease in IgA nephropathy. Clin Proteomics, 2017. 14 : p. 30.

22. Sargsyan, S.A., et al., Detection of glomerular complement C3 fragments by magnetic resonance imaging in murine lupus nephritis.Kidney Int, 2012. 81 (2): p. 152-9.

23. Martinez-Barricarte, R., et al., Human C3 mutation reveals a mechanism of dense deposit disease pathogenesis and provides insights into complement activation and regulation. J Clin Invest, 2010.120 (10): p. $3702-12$.

24. Hilhorst, M., et al., Complement in ANCA-associated glomerulonephritis. Nephrol Dial Transplant, 2017. 32 (8): p. 1302-1313. 
25. Panzer, S.E., et al., Glomerular C3 Deposition Is an Independent Risk Factor for Allograft Failure in Kidney Transplant Recipients With Transplant Glomerulopathy. Kidney Int Rep, 2019.4 (4): p. 582-593.

26. Ohsawa, I., et al., Extraglomerular C3 deposition and metabolic impacts in patients with IgA nephropathy. Nephrol Dial Transplant, 2013. 28 (7): p. 1856-64.

27. Nam, K.H., et al., Predictive value of mesangial C3 and C4d deposition in IgA nephropathy. Clin Immunol, 2020. 211 : p. 108331.

28. Kim, S.J., et al., Decreased circulating C3 levels and mesangial C3 deposition predict renal outcome in patients with IgA nephropathy. PLoS One, 2012. 7 (7): p. e40495.

Figure and table legends

FIGURE 1. Study scheme. The patients were selected into or excluded from the study according to the scheme. Representative photographs of C3 immunofluorescence staining. Bar=50um.

FIGURE 2. Glomerular immunodeposits from the patients in $\mathrm{C}^{\text {low }}$ and $\mathrm{C3}^{\text {high }}$ group. (A, B, D, E, G and $\mathbf{H}$ ) Representative photographs of glomerular immune complexes deposition (IgA, IgG and $\mathrm{IgM}$ ) from $\mathrm{C} 3^{\text {low }}$ and $\mathrm{C} 3^{\text {high }}$ group. Bar $=50 \mu \mathrm{m}$. (C, F and I)The difference of glomerular immune complexes deposition (IgA, IgG and IgM) in $\mathrm{C} 3^{\text {low }}$ and $\mathrm{C} 3^{\text {high }}$ group. Glomerular IgA deposition was graded by $\operatorname{IgA}=2+$ and $\operatorname{IgA}>2+$; Glomerular IgG, IgM deposition was graded by absent (score, 0), mild (score, 1), severe (score, 2), respectively. The difference of constituent ratio was analyzed by Chi square test.

FIGURE 3. Morphological characteristics of the biopsies from the patients in $\mathrm{C3}^{\text {low }}$ and $\mathrm{C3}^{\text {high }}$ group.(A-F) Representative photographs of PAS staining, Masson staining and electron microscopy from $\mathrm{C} 3^{\text {low }}$ and $\mathrm{C} 3^{\text {high }}$ group. Bar $=50 \mu \mathrm{m}$. The magnification by electron microscopy was 2000 times.

FIGURE 4. $\mathrm{C3}^{\text {high }}$ group contained more lymphocytes in $\mathrm{C3}^{\text {low }}$ group in IgAN renal biopsies.A, B, D, E, G, $\mathbf{H}$ represent photographs of $\mathrm{CD} 68^{+}, \mathrm{CD} 4^{+}$and $\mathrm{CD} 8^{+}$cells in $\mathrm{C} 3^{\text {low }}$ and $\mathrm{C} 3^{\text {high }}$ groups. $\mathbf{C}, \mathbf{F}, \mathbf{I}$ showed renal tissues were revealed more $\mathrm{CD} 68^{+}, \mathrm{CD} 4^{+}$and $\mathrm{CD} 8^{+}$cells infiltration in the $\mathrm{C} 3^{\text {high }}$ group. Box plots indicated statistical differences. Values greater than $95 \%$ or less than $5 \%$ is listed as a dot while values less than $95 \%$ or greater than $5 \%$ were hidden in positive and negative error line. The line in the center of each box represents the median value, and the upper and lower ends of the box are the upper and lower quartiles, Bar $=50 \mu \mathrm{m}$.

FIGURE 5. Renal survival curve of creatinine doubling and ESRD. (A) Kaplan-Meier estimates of proportion of renal survival (absence of creatinine doubling) in $83.3 \%(85 / 102)$ v.s. $61.8 \%(21 / 34)$ of the patients in $\mathrm{C} 3^{\text {low }}$ and $\mathrm{C} 3^{\text {high }}$ group, respectively. Estimated overall survival time were 97.8 (95\% CI:93.4102.2) months for patients in C3low group; 81.9 (95\% CI: 70.7-93.0) months for patients in C3 ${ }^{\text {high }}$ group, $\mathrm{P}=0.025$ (log rank test). (B) Kaplan-Meier estimates of proportion of renal survival (absence of ESRD) in $93.1 \%(95 / 102)$ v.s. $67.6 \%(23 / 34)$ of the patients in $\mathrm{C} 3^{\text {low }}$ and $\mathrm{C} 3^{\text {high }}$ group, respectively. Estimated survival time were 100.2 (95\% CI: 96.4-104.1) months for patients in C3 ${ }^{\text {low }}$ group; 86.5 (95\% CI: 75.9-97.0) months for patients $\mathrm{C} 3{ }^{\text {high }}$ group, $\mathrm{P}=0.002$ (log rank test).

Table 1. Demographic, clinical and biochemical characteristics between patients with low or high levels of glomerular C3 deposition

SUPPLEMENTARY FIGURE. Renal tissues were revealed more interstitial tryptase ${ }^{+}$mast cells infiltration in the $\mathrm{C}^{\text {high }}$ group in renal biopsies of IgAN patients rather than $\mathrm{CD20}^{+} \mathrm{B}$ lymphocytes and $\mathrm{CD}^{+} \mathrm{c}^{+}$myeloid dendritic cells. Box plots indicated statistical differences. Values greater than $95 \%$ or less than $5 \%$ is listed as a dot while values less than $95 \%$ or greater than $5 \%$ were hidden in positive and negative error line. The line in the center of each box represents the median value, and the upper and lower ends of the box are the upper and lower quartiles. 
Figure 1.

A

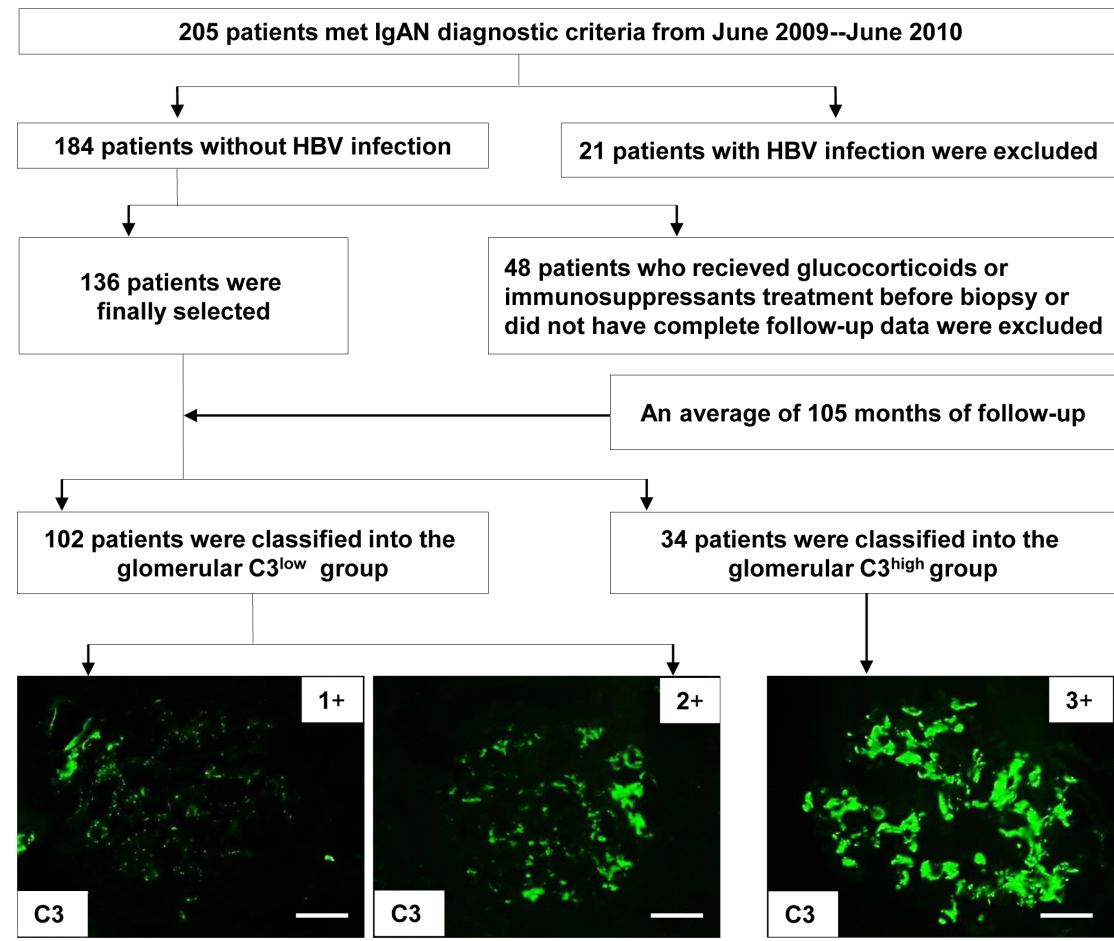

Figure 2.
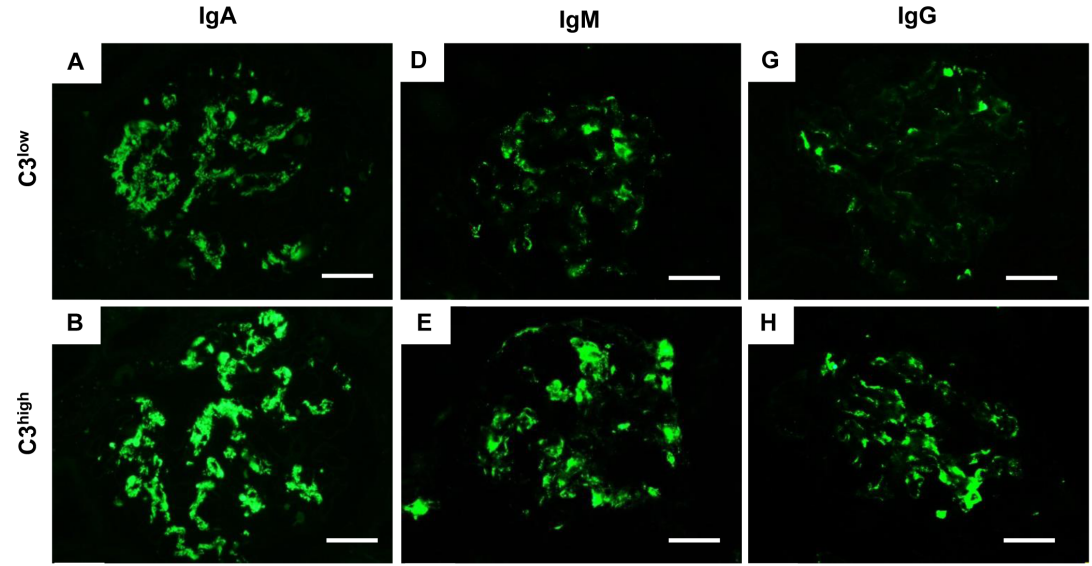

$$
\text { C }
$$

F

Glomerular IgA deposition

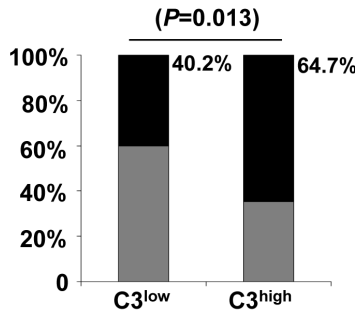

Glomerular IgM deposition

I

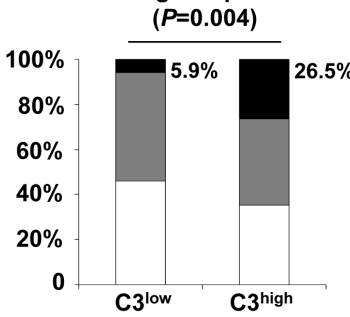

$\square \lg A=2+\square \lg A>2+$

IgM score $\square 0 \square 1 \square 2$

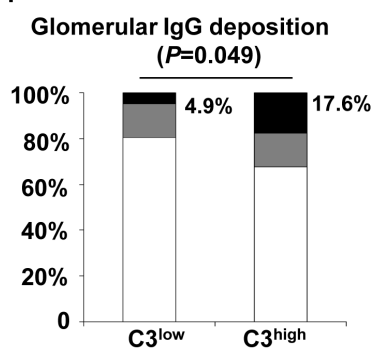

IgG score $\square 0 \square 1 \square 2$ 
Figure 3.

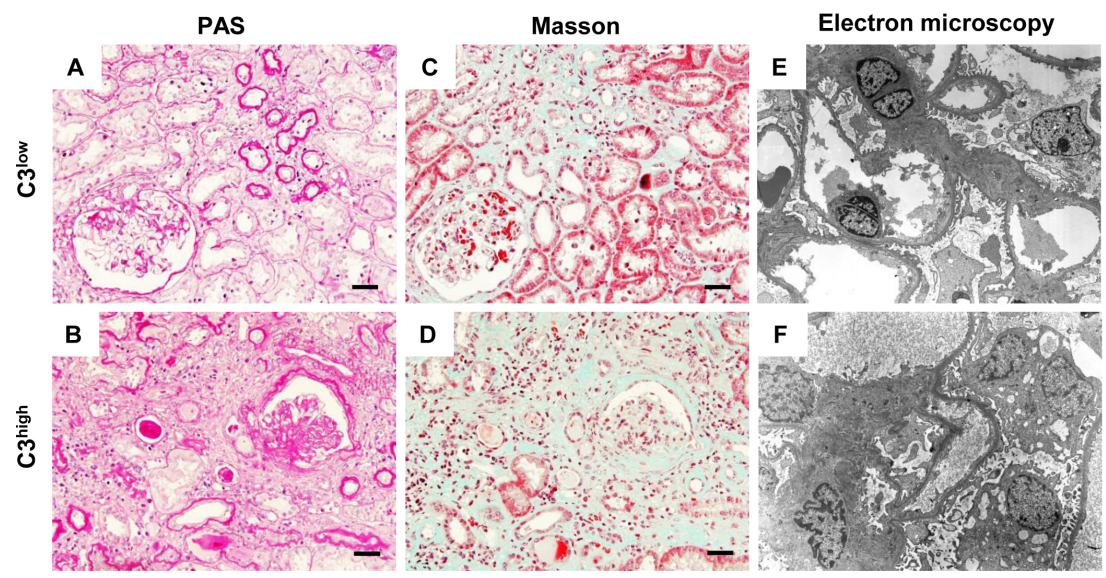

Figure 4.
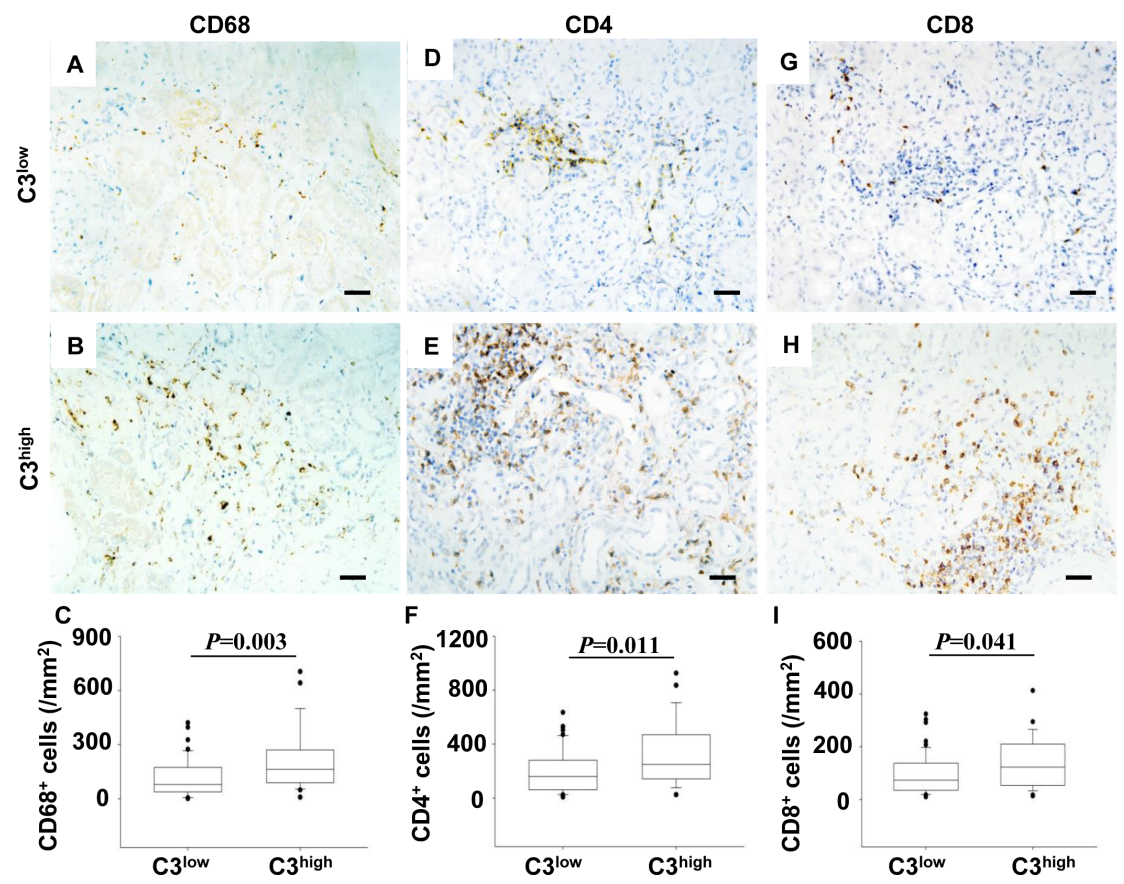
Figure 5.
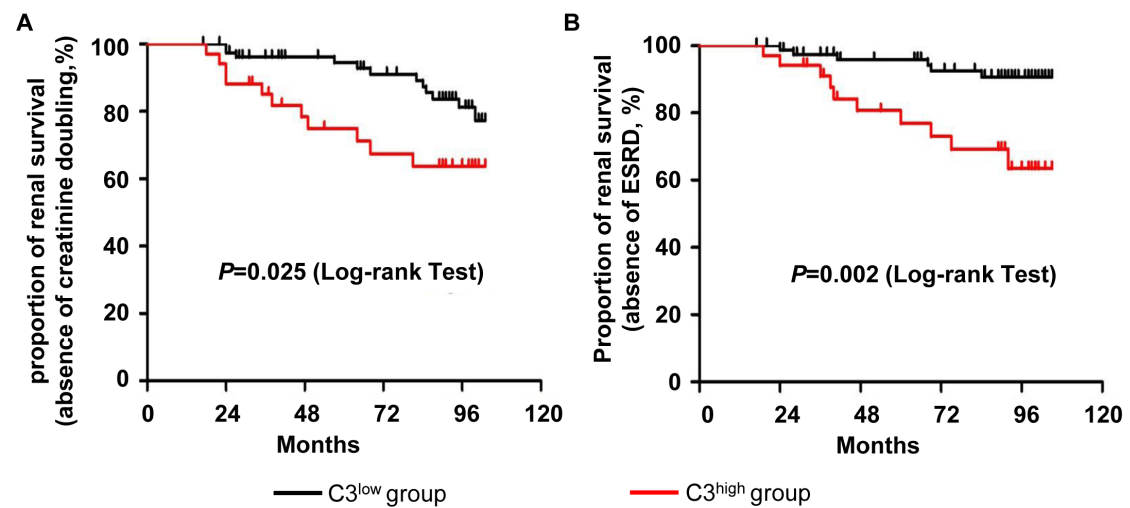

\section{Hosted file}

table.doc available at https://authorea.com/users/340212/articles/467332-severe-glomerularc3-deposition-indicated-severe-renal-lesions-and-poor-prognosis-in-patients-withimmunoglobulin-a-nephropathy 